\title{
Correction to: High-Resolution Colonic Manometry Pressure Profiles Are Similar in Asymptomatic Diverticulosis and Controls
}

\author{
Rebekah Jaung ${ }^{1} \cdot$ Chris Varghese ${ }^{1} \cdot$ Anthony Y. Lin $^{1} \cdot$ Niranchan Paskaranandavadivel $^{2} \cdot$ Peng $^{D^{2}}{ }^{2}$. \\ David Rowbotham $^{3} \cdot$ Phil Dinning ${ }^{4,5} \cdot$ Gregory O'Grady $^{1,2} \cdot$ Ian Bissett $^{1}$
}

Published online: 8 August 2020

๑) Springer Science+Business Media, LLC, part of Springer Nature 2020

\section{Correction to: Digestive Diseases and Sciences https://doi.org/10.1007/s10620-020-06320-4}

The original version of the article unfortunately contained an error in the unit used for 'Distance of propagation'. The distance of propagation should be in the unit ' $\mathrm{cm}$ ' rather than 'mm' throughout the manuscript.

In the Abstract, under 'Methods' subsection, the sentence 'Antegrade and retrograde propagating contractions, distance

The original article can be found online at https://doi.org/10.1007/ s10620-020-06320-4.

Electronic supplementary material The online version of this article (https://doi.org/10.1007/s10620-020-06524-8) contains supplementary material, which is available to authorized users.

Gregory O'Grady

greg.ogrady@auckland.ac.nz

Rebekah Jaung

rebekahjaung@gmail.com

Chris Varghese

cvar706@aucklanduni.ac.nz

Anthony Y. Lin

drtlin@gmail.com

Niranchan Paskaranandavadivel nira.pask@auckland.ac.nz

Peng Du

peng.du@auckland.ac.nz

David Rowbotham

DavidRB@adhb.govt.nz

Phil Dinning

phil.dinning@flinders.edu.au of propagation (mm)...' should read, 'Antegrade and retrograde propagating contractions, distance of propagation (cm)...'

Tables 1, 2 and Figure 3 with the corrected unit for 'Distance of Propagation' is given below. The unit is updated in the ESM files as well.

Ian Bissett

i.bissett@auckland.ac.nz

1 Department of Surgery, Faculty of Medical and Health Sciences, University of Auckland, Private Bag 92019, Auckland 1142, New Zealand

2 Auckland Bioengineering Institute, University of Auckland, Private Bag 92019, Auckland 1142, New Zealand

3 Department of Gastroenterology and Hepatology, Auckland City Hospital, 2 Park Road, Grafton, Auckland 1023, New Zealand

4 Discipline of Human Physiology, Flinders University, Adelaide, SA, Australia

5 Department of Gastroenterology, Flinders Medical Centre, Bedford Park, SA 5042, Australia 
Table 1 Colonic motility patterns in the descending colon

\begin{tabular}{|c|c|c|c|c|c|c|}
\hline \multirow[t]{2}{*}{ Descending colon } & \multicolumn{3}{|c|}{ Control $(n=9)$} & \multicolumn{3}{|c|}{ Diverticulosis $(n=9)$} \\
\hline & Premeal & Post-meal & $p$ & Premeal & Post-meal & $p$ \\
\hline \multicolumn{7}{|l|}{ All activity ${ }^{\mathrm{a}}$} \\
\hline Total no. of propagating contractions & $7.3(2.5)$ & $64.3(22)$ & 0.004 & $11.8(4.7)$ & 46.8 (13) & 0.012 \\
\hline No. of antegrade propagating contractions & $2.6(1.5)$ & $11.2(3.1)$ & $\mathbf{0 . 0 2 1}$ & $0.6(0.3)$ & $6.1(3.0)$ & 0.012 \\
\hline No. of retrograde propagating contractions & $4.0(1.4)$ & $50.6(19)$ & 0.0039 & $8.6(4.4)$ & $27.0(11)$ & 0.09 \\
\hline Mean distance propagated $(\mathrm{cm})$ & $13.9(4.2)$ & $20.0(2.0)^{\mathrm{c}}$ & 0.25 & $7.3(2.8)$ & $10.8(1.5)^{\mathrm{c}}$ & 0.17 \\
\hline Mean amplitude $(\mathrm{mmHg})$ & $34.2(9.6)$ & $48.7(5.8)$ & 0.091 & $21.7(6.8)$ & $32.3(5.7)$ & 0.079 \\
\hline \multicolumn{7}{|l|}{ Cyclic motor patterns ${ }^{\mathrm{b}}$} \\
\hline Total no. of CMPs & - & $32.44(19)$ & 0.014 & - & $16.44(7.9)$ & 0.10 \\
\hline No. of antegrade CMPs & - & $2.89(2.9)$ & 1 & - & $2.11(2.0)$ & 0.37 \\
\hline No. of retrograde CMPs & - & $26.00(15)$ & 0.022 & - & $12.78(7.3)$ & 0.10 \\
\hline Mean distance propagated of CMPs $(\mathrm{cm})$ & - & $17.08(2.8)^{\mathrm{c}}$ & 0.0003 & - & $6.04(2.5)^{\mathrm{c}}$ & 0.10 \\
\hline Mean amplitude of CMPs (mmHg) & - & $37.51(7.2)$ & 0.0008 & - & $20.04(8.0)$ & 0.10 \\
\hline
\end{tabular}

${ }^{a}$ Comparing magnitude of meal response $(\Delta)$ between controls and diverticulosis patients: number of propagating contractions, $p=0.45$; antegrade, $p=0.14$; retrograde, $p=0.085$; amplitude, $p=0.82$; distance, $p=0.65$

${ }^{\mathrm{b}}$ No premeal CMPs therefore magnitude of difference $(\Delta)$ is equal to post-meal responses

${ }^{c} p \leq 0.01$ difference between controls and diverticulosis participants

Bold values indicate statistically significance $p<0.05$

Table 2 Colonic motility patterns in the sigmoid colon

\begin{tabular}{|c|c|c|c|c|c|c|}
\hline \multirow[t]{2}{*}{ Sigmoid colon } & \multicolumn{3}{|c|}{ Control $(n=9)$} & \multicolumn{3}{|c|}{ Diverticulosis $(n=9)$} \\
\hline & Premeal & Post-meal & $p$ & Premeal & Post-meal & $p$ \\
\hline \multicolumn{7}{|l|}{ All activity ${ }^{\mathrm{a}}$} \\
\hline Total no. of propagating contractions & $9.9(3.4)$ & $79.2(18)$ & 0.0051 & $11.8(4.7)$ & $46.8(13)$ & 0.012 \\
\hline No. of antegrade propagating contractions & $2.6(1.1)$ & $19.2(9.2)$ & $\mathbf{0 . 0 3 9}$ & $0.6(0.3)$ & $6.1(3.0)$ & 0.0012 \\
\hline No. of retrograde propagating contractions & $5.9(2.6)$ & $54.1(12)$ & 0.0032 & $8.6(4.4)$ & $27.0(11)$ & 0.090 \\
\hline Mean distance propagated $(\mathrm{cm})$ & $12.2(3.3)$ & $20.2(5.9)^{\mathrm{c}}$ & 0.34 & $7.3(2.8)$ & $10.8(1.5)^{\mathrm{c}}$ & 0.17 \\
\hline Mean amplitude $(\mathrm{mmHg})$ & $40.0(9.0)$ & $46.9(5.4)$ & 0.34 & $19.3(6.5)$ & $32.3(5.7)$ & 0.040 \\
\hline \multicolumn{7}{|l|}{ Cyclic motor patterns ${ }^{\mathrm{b}}$} \\
\hline Total no. of CMPs & $0.9(0.9)$ & $50.2(18)$ & 0.024 & - & $16.9(11)$ & 0.18 \\
\hline No. of antegrade CMPs & - & $9.2(7.9)$ & 0.10 & - & $2.6(2.2)$ & 0.18 \\
\hline No. of retrograde CMPs & $0.6(0.6)$ & $35.8(11)$ & 0.011 & - & $13.9(9.6)$ & 0.18 \\
\hline Mean distance propagated of CMPs $(\mathrm{cm})$ & $0.6(0.6)$ & $9.5(2.0)$ & 0.0019 & - & $4.8(2.5)$ & 0.18 \\
\hline Mean amplitude of CMPs (mmHg) & $2.7(2.7)$ & $33.0(7.8)$ & 0.0082 & - & $17.3(8.7)$ & 0.18 \\
\hline
\end{tabular}

${ }^{\mathrm{a}}$ Comparing magnitude of meal response $(\Delta)$ between controls and diverticulosis patients: number of propagating contractions, $p=0.13$; antegrade, $p=0.5$; retrograde, $p=\mathbf{0 . 0 4 2}$; amplitude, $p=0.56$; distance, $p=0.6$

${ }^{\mathrm{b}}$ Comparing magnitude of meal response $(\Delta)$ of CMPs between controls and diverticulosis patients: number of propagating contractions, $p=0.079 ;$ antegrade, $p=0.43$; retrograde, $p=0.071$; amplitude, $p=0.41$; distance, $p=0.23$

${ }^{c} p=0.01$ difference between controls and diverticulosis participants

Bold values indicate statistically significance $p<0.05$ 

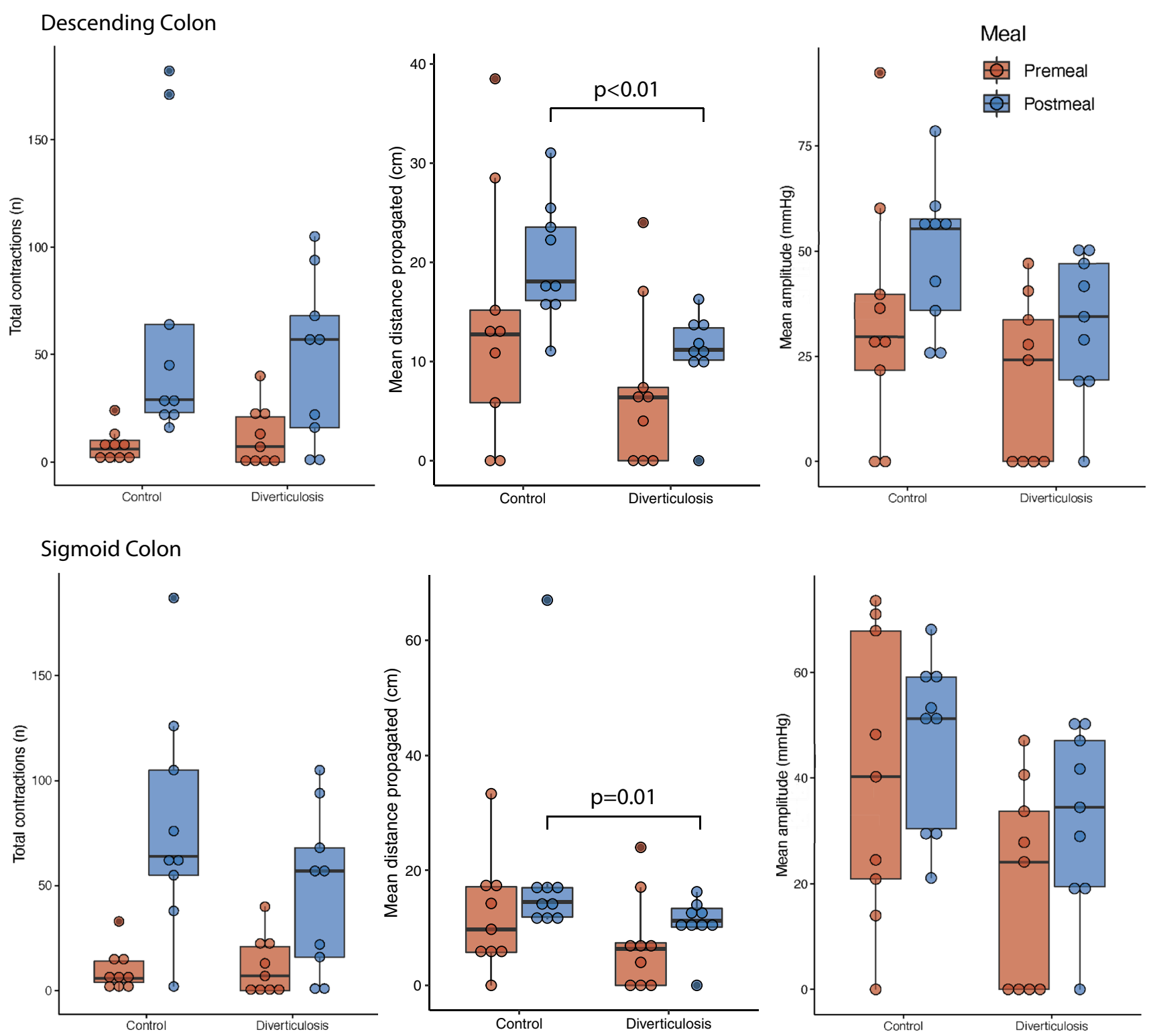

Fig. 3 Individual characteristics of propagating contractions of control and diverticulosis participants. Top row $=$ descending colon, bottom row $=$ sigmoid colon

Publisher's Note Springer Nature remains neutral with regard to jurisdictional claims in published maps and institutional affiliations. 